\title{
High Resolution Spectroscopy of 4U 1728-34 from a Simultaneous Chandra-RXTE Observation
}

\author{
A. D’ Aí*, T. Di Salvo*, R. Iaria*, G. Lavagetto*, N. R. Robba*, L. Burderi ${ }^{\dagger}$, \\ M. Mendez ${ }^{* *}$ and M. van der Klis* \\ ${ }^{*}$ Dipartimento di Scienze Fisiche ed Astronomiche, Università di Palermo, via Archirafi n.36, \\ 90123 Palermo, Italy. \\ Osservatorio Astronomico di Roma, Via Frascati 33, 00040 Monteporzio Catone (Roma), Italy. \\ ${ }^{* *}$ SRON National Institute for Space Research, Sorbonnelaan 2, 3584 CA Utrecht, the Netherlands \\ "Astronomical Institute "Anton Pannekoek," University of Amsterdam and Center for High-Energy \\ Astrophysics, Kruislaan 403, NL 1098 SJ Amsterdam, the Netherlands
}

\begin{abstract}
We report on a simultaneous Chandra and RossiXTE observation of the LMXB atoll bursting source $4 \mathrm{U} 1728-34$ performed on 2002 March 3-5. We fitted the $1.2-35 \mathrm{keV}$ continuum spectrum with a blackbody plus a Comptonized component. An overabundance of Si by a factor of $\sim 2$ with respect to Solar abundance is required for a satisfactory fit. Large residuals at 6-10 keV can be fitted by a broad (FWHM $\simeq 1.6 \mathrm{keV}$ ) Gaussian emission line, or, alternatively, by absorption edges associated with $\mathrm{Fe} \mathrm{I}$ and $\mathrm{Fe} \mathrm{XXV}$ at $\sim 7.1 \mathrm{keV}$ and $\sim 9 \mathrm{keV}$, respectively. In this interpretation, we find no evidence of broad, or narrow $\mathrm{Fe} \mathrm{K} \alpha$ line, between 6 and $7 \mathrm{keV}$. We tested our alternative modeling of the iron $\mathrm{K} \alpha$ region by reanalyzing a previous BeppoSAX observation of $4 \mathrm{U}$ 1728-34, finding a general agreement with our new spectral model.
\end{abstract}

Keywords: accretion discs - stars: individual: $4 \mathrm{U}$ 1728-34 — stars: neutron stars - X-ray: stars - X-ray: spectrum - X-ray: general

PACS: $97.80 . \mathrm{Jp}$

\section{INTRODUCTION}

$4 \mathrm{U} 1728-34$ is a well known prototype of the class of the bursting atoll sources ([4]). This was one of the first sources to display $\mathrm{kHz}$ QPOs in its power spectrum and the first one to display burst oscillations, around $363 \mathrm{~Hz}$, during some type I X-ray bursts ([7]). Its temporal behavior has been recently extensively studied ([2], [10]) using a large set of RXTE observations, spanning more than three years. Spectral studies have been carried out in the past with the use of EXOSAT ([11]), RXTE ([6]) and BeppoSAX ([1], [6]) satellites; although there was a general agreement about the modelization of the continuum emission, it was disputed the nature of local features, especially a broad Gaussian line at 6.2-6.7 keV. The distance to the source is estimated between $4.1 \mathrm{kpc}$ and $5.1 \mathrm{kpc}([3] ;$ [1]).

In this paper we present the results from a simultaneous Chandra-RXTE observation of $4 \mathrm{U}$ 1728-34. We propose an alternative fit of the iron K-shell region, using two absorption edges instead of a broad emission line, whose interpretation would be quite problematic. 


\section{OBSERVATIONS AND SPECTRAL ANALYSIS}

4U 1728-34 was observed by Chandra on 2002 March 4 for a total collecting time of $\sim 30 \mathrm{ksec}$. We used the Chandra High Energy Transmission Grating Spectrometer (HETGS) to perform a high resolution spectroscopic analysis. The data were collected in the Timed Exposure Mode, and a sub-array was adopted $(\mathrm{q}=1$ and $\mathrm{n}=400$, see the Chandra Proposer's Observatory Guide at http://cxc.harvard.edu/proposer/POG) in order to mitigate the effects of photon pile-up for first order spectra. Consequently the frame time was $1.44 \mathrm{~s}$, the High Energy Grating (HEG) spectrum is cut below $1.6 \mathrm{keV}$ and the Medium Energy Grating (MEG) spectrum below $1.2 \mathrm{keV}$. No systematic error was added to the data. The RXTE observation started on 2002 March 3 03:27:12 and ended on 2002 March 5 13:00:00. For the spectral analysis we used only the Proportional Counter Units 2 and 3 data in the Standard 2 configuration (with 16 s time resolution and 129 energy channels). Seven type-I bursts were revealed in the PCA lightcurve and two bursts in the Chandra lightcurve. As our primary concern is to focus on the persistent emission of the source we discarded data around each burst, for a time length of $160 \mathrm{~s}$.

As concerns the Chandra HETG data, we considered the four first-order dispersed spectra, namely the two HEG spectra and the two MEG spectra on the opposite sides of the zeroth order. We averaged HEG+1 (MEG+1) and HEG-1 (MEG-1) spectra in a single spectrum, after having tested their reciprocal consistency. The used energy range is $1.6-10 \mathrm{keV}$ for the HEG spectrum and 1.2-5 keV for the MEG spectrum. For all the fits we took into account an instrumental feature at $2.07 \mathrm{keV}$ for bright sources (described by [5]) and fit it with an inverse edge (with optical depth $\tau \simeq-0.1$ ). The HEG and MEG spectra were binned in order to have at least 300 counts for each bin. This, however, still ensures a high number of channels (about 1000) and good spectral resolution throughout the entire covered energy band. Concerning the RXTE/PCA data the standard selection criteria for obtaining the Good Time Intervals were applied. We restricted the spectral analysis to the temporal intervals during which RXTE operated simultaneously with Chandra. We limited the energy range to $3.5-35 \mathrm{keV}$, and applied a $2 \%$ systematic error for channels below $25 \mathrm{keV}$ and $2.5 \%$ for channels above $25 \mathrm{keV}$. Relative normalizations of the three instruments, except for HEG which was fixed to a reference value of 1, were left as free parameters in all the fits performed.

We tried a series of models to simultaneously fit HEG, MEG and RXTE spectra. We found the best-fit model to consist of a soft emission, described by a blackbody of temperature $\simeq 0.52 \mathrm{keV}$, plus a Comptonized component (CompTT in XSPEC, [8]), where the seed photon temperature $\mathrm{kT}_{0}$ is $\simeq 1.3 \mathrm{keV}$, the electron temperature $\mathrm{kT}_{e}$ is $\simeq 7.4 \mathrm{keV}$ and, finally, the optical depth $\tau$ associated to a spherical corona is $\simeq 6.2$. Both components are photo-electrically absorbed by an equivalent hydrogen column $N_{H} \simeq 2.3 \times 10^{22} \mathrm{~cm}^{-2}$. The associated $\chi^{2} /$ d.o.f. obtained for this fit is $1206 / 1034$. We noted an evident absorption edge around $1.84 \mathrm{keV}$, probably associated to neutral $\mathrm{Si}$, in the MEG and HEG spectra. To fit this edge we substituted the component phabs in XSPEC with the component vphabs, which allows us to vary the abundances of single elements with respect to the solar abundances. We found that leaving the Si abundance free improves the fits significantly; Si resulted overabundant by a factor $\sim 2$ with respect to the solar abundance $\left(\chi^{2} /\right.$ d.o.f. value obtained for this fit is $\left.1031 / 1033\right)$.

Large residuals at $6-10 \mathrm{keV}$ can be fitted by a very broad Gaussian emission line 
$(\sigma \simeq 0.7 \mathrm{keV}$, corresponding to a FWHM $\simeq 1.6 \mathrm{keV}$ ), whose interpretation is quite problematic, or, alternatively, by absorption edges associated with Fe I and Fe XXV at $7.03 \mathrm{keV}(\tau \simeq 0.11)$ and $\sim 9 \mathrm{keV}$ ( $\tau \simeq 0.16$, respectively. The addition of these edges improves the fit significantly compared to the simple model described above (giving a decrease of $\chi^{2} /$ d.o.f. from $1031 / 1033$ to 959/1029). In this interpretation, we find no evidence of broad, or narrow Fe K $\alpha$ line, between 6 and $7 \mathrm{keV}$. To test the consistency of our model we reanalyzed a previous BeppoSAX observation performed between August 23 and 24, 1998 (see [1]). A Gaussian emission line is no longer statistically required if we introduce two absorption edges at energies above $7 \mathrm{keV}$. We found a first edge at $\simeq 7.4 \mathrm{keV}(\tau \simeq 0.08)$ and a second edge at $\simeq 8.7 \mathrm{keV}(\tau \simeq 0.06)$. We found a general agreement with the Chandra-RXTE spectrum, obtaining $\chi^{2} /$ d.o.f. $=225 / 179$ (instead of $\chi^{2} /$ d.o.f. $=236 / 178$ obtained for the model adopted in [1]). In table 1 and Figure 1 we present the results of the two fits of the BeppoSAX and Chandra-RXTE datasets.

\section{CONCLUSIONS}

We confirm that the best-fit continuum model for this source is composed of two components: a blackbody emission which probably comes from the inner edge of an accretion disk around the compact object, and a Comptonized component coming from a hot corona surrounding the system. Although we cannot definitely exclude that a quite broad Gaussian line $(\sigma \simeq 0.7 \mathrm{keV}, \mathrm{FWHM} \simeq 1.6 \mathrm{keV})$ is present in this source, we present here a different interpretation of the residuals in the iron K-shell region. These are well fitted by two absorption edges at energies at $\sim 7$ and $\sim 9 \mathrm{keV}$, associated with Fe I and Fe XXV, respectively (see e.g. [9]). We derived, however, an upper limit to the flux of a broad emission line, fixing the values of energy and width to the values found in [1]; we find an upper limit to the line flux of $1.7 \times 10^{-11} \mathrm{erg} \mathrm{cm}^{-2} \mathrm{sec}^{-1}$ (corresponding to an equivalent width of $28 \mathrm{eV}$ ).

We checked that this alternative model is also in agreement with a previous BeppoSAX observation. We observe a shift in the energy of the Fe I edge from $7.1 \mathrm{keV}$ during the Chandra-RXTE observation to $7.4 \mathrm{keV}$ (compatible with $\mathrm{K} \alpha$ edges of moderately ionized iron, Fe IX to Fe XVI) during the BeppoSAX observation, while the energy of the Fe XXV edge is consistent with being the same in both observations. Finally we found, both in the Chandra-RXTE and BeppoSAX spectra, a Si overabundance by a factor $\sim 2-2.5$ with respect to the solar abundance and the values obtained from the fit are consistently in agreement.

\section{REFERENCES}

1. Di Salvo T., Iaria R., Burderi L., Robba N.R., 2000, ApJ, 542, 1034

2. Di Salvo T., Mendez M., van der Klis M., Ford E., Robba N.R., 2001, ApJ, 546, 1107

3. Galloway D.K., Psaltis D., Chakrabarty D., Muno M.P., 2003, ApJ, 590, 999

4. Hasinger, G., \& van der Klis, M., 1989, A\&A, 225, 79

5. Miller J.M., Fabian A.C., Wijnands R., et al., 2002, ApJ, 578, 450

6. Piraino S., Santangelo A., Kaaret P., 2000, A\&A, 360, L35

7. Strohmayer T.E., Zhang W., Swank J.H., et al., 1996, ApJ, 469, L9

8. Titarchuck L., 1994, ApJ, 434, 570 
TABLE 1. Best fit parameters for the two datasets of $4 \mathrm{U} 1728-34$, obtained from a BeppoSAX observation (0.12-60 keV energy band) and a joint CHANDRA-RXTE observation (1.2-35 keV energy band). The continuum emission consists of a thermal blackbody (bbody) and a Comptonized component modeled by compTT. $k T_{\mathrm{BB}}$ and $\mathrm{N}_{\mathrm{BB}}$ are, respectively, the blackbody temperature and normalization in units of $L_{39} / D_{10}^{2}$, where $L_{39}$ is the luminosity in units of $10^{39} \mathrm{ergs} / \mathrm{s}$ and $D_{10}$ is the distance in units of $10 \mathrm{kpc} . k T_{0}$, $k T_{\mathrm{e}}$ and $\tau$ indicate the seed-photon temperature, the electron temperature and the optical depth of the Comptonizing cloud around the neutron star. $\mathrm{N}_{\mathrm{Comptt}}$ is the normalization of the Comptt model in XSPEC v.11.2.0 units. Unabsorbed luminosities of the bbody component and of the CompTT component are calculated assuming a distance to the source of $5.1 \mathrm{kpc}$ ([1]). For the component Edge, $\mathrm{E}_{\text {edge }}$ denotes the energy of the edge and $\tau$ the optical depth. Uncertainties are at $90 \%$ confidence level for a single parameter.

\begin{tabular}{|c|c|c|c|}
\hline & & BeppoSAX & Chandra - RXTE \\
\hline Component & Parameter (Units) & \multicolumn{2}{|c|}{ Values } \\
\hline vpha & $N_{\mathrm{H}}\left(10^{22} \mathrm{~cm}^{-2}\right)$ & $2.37_{-0.11}^{+0.14}$ & $2.61_{-0.07}^{+0.06}$ \\
\hline vpha & Si (Solar units) & $2.1_{-0.5}^{+0.5 .11}$ & $2.02_{-0.13}^{+0.13}$ \\
\hline edge & $\mathrm{E}_{\text {edge }}(\mathrm{keV})$ & $7.41_{-0.14}^{+0.15}$ & $7.03_{-0.06}^{+0.08}$ \\
\hline edge & $\tau\left(10^{-2}\right)$ & $8_{-2}^{+2}$ & $11_{-4}^{+3}$ \\
\hline edge & $\mathrm{E}_{\text {edge }}(\mathrm{keV})$ & $8.73_{-0.24}^{+0.26}$ & $9.0_{-0.4}^{+0.3}$ \\
\hline edge & $\tau\left(10^{-2}\right)$ & $6_{-3}^{+3}$ & $16_{-4}^{+4}$ \\
\hline bbody & $\mathrm{kT}(\mathrm{keV})$ & $0.573_{-0.024}^{+0.002}$ & $0.516_{-0.014}^{+0.002}$ \\
\hline bbody & $\mathrm{N}_{\mathrm{BB}}\left(10^{-3}\right)$ & $21.5_{-1.3}^{+1.0 .024}$ & $10.3_{-0.4}^{+0.4}$ \\
\hline bbody & Luminosity $\left(10^{36} \mathrm{erg} \mathrm{cm}^{-2} \mathrm{sec}^{-1}\right)$ & $5.7^{-1.3}$ & $0.87^{-0.4}$ \\
\hline CompTT & $k T_{0}(\mathrm{keV})$ & $1.53_{-0.07}^{+0.06}$ & $1.33_{-0.05}^{+0.05}$ \\
\hline CompTT & $k T_{\mathrm{e}}(\mathrm{keV})$ & $6.4_{-0.4}^{+1.3 .07}$ & $7.4_{-0.4}^{+0.500}$ \\
\hline CompTT & $\tau$ & $4.8_{-1.0}^{+0.4}$ & $6.2_{-0.6}^{+0.4}$ \\
\hline CompTT & $\mathrm{N}_{\text {CompTT }}\left(10^{-2}\right)$ & $6.8_{-1.4}^{+1.3}$ & $4.4_{-0.6}^{+0.3}$ \\
\hline \multirow[t]{2}{*}{ CompTT } & Luminosity $\left(10^{36} \mathrm{erg} \mathrm{cm}^{-2} \mathrm{sec}^{-1}\right)$ & $10.2^{-1.4}$ & $2.6^{-0.6}$ \\
\hline & $\chi^{2} /$ dof & $225 / 179$ & $959 / 1029$ \\
\hline
\end{tabular}
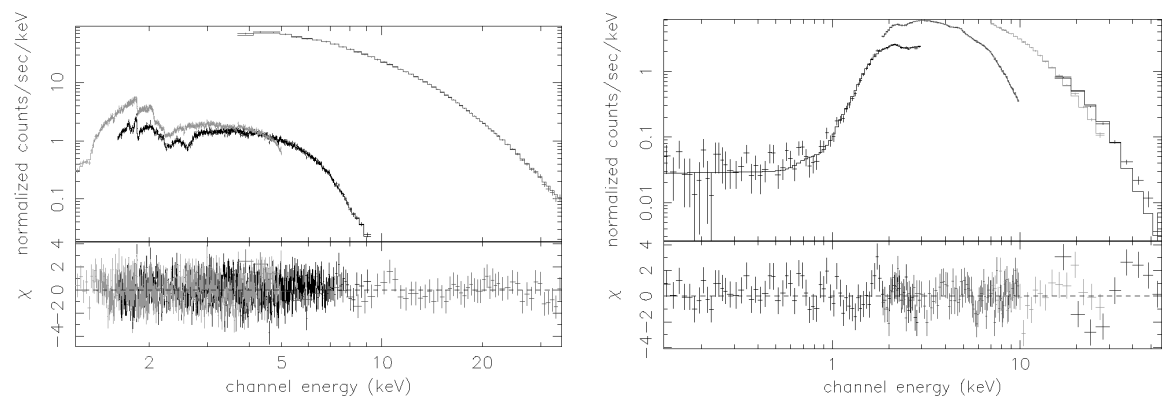

FIGURE 1. Spectra of 4U 1728-34 shown together with the best-fit model (see Table 1). Left panel: 1.2-35.0 keV spectrum obtained from the simultaneous Chandra and RXTE dataset. Right panel: 0.12$60 \mathrm{keV}$ spectrum obtained from a BeppoSAX observation. In smaller panels: residuals in unit of $\sigma$ with respect to the best-fit model.

9. Turner T.J., Done C., Mushotzky R., Madejski G., Kunieda H., 1992, ApJ, 391, 102

10. van Straaten S., van der Klis M., Di Salvo T., Belloni T., 2002, ApJ, 568, 912

11. White N.E., Peacock A., Hasinger G., et al., 1986, MNRAS, 218, 129 